\title{
Dense deformation field estimation for atlas-based segmentation of pathological MR brain images
}

\author{
M. Bach Cuadra ${ }^{a, *}$, M. De Craene ${ }^{b}$, V. Duay ${ }^{a}$, B. Macq ${ }^{b}$, C. Pollo ${ }^{a, c}$, J.-Ph. Thiran ${ }^{a}$ \\ a Signal Processing Institute (ITS), École Polytechnique Fédérale de Lausanne (EPFL), CH-1015 Lausanne, Switzerland \\ b Communications Laboratory, Université Catholique de Louvain (UCL), B-1348, Louvain-la-Neuve, Belgium \\ c Department of Neurosurgery, Lausanne University Hospital (CHUV), CH-1011, Lausanne, Switzerland
}

\section{A R T I C L E I N F O}

Article history:

Received 9 May 2006

Received in revised form

8 August 2006

Accepted 8 August 2006

Keywords:

Atlas-based segmentation

Brain tumors

MR imaging

Registration

\begin{abstract}
A B S T R A C T
Atlas registration is a recognized paradigm for the automatic segmentation of normal MR brain images. Unfortunately, atlas-based segmentation has been of limited use in presence of large space-occupying lesions. In fact, brain deformations induced by such lesions are added to normal anatomical variability and they may dramatically shift and deform anatomically or functionally important brain structures. In this work, we chose to focus on the problem of inter-subject registration of MR images with large tumors, inducing a significant shift of surrounding anatomical structures. First, a brief survey of the existing methods that have been proposed to deal with this problem is presented. This introduces the discussion about the requirements and desirable properties that we consider necessary to be fulfilled by a registration method in this context: To have a dense and smooth deformation field and a model of lesion growth, to model different deformability for some structures, to introduce more prior knowledge, and to use voxel-based features with a similarity measure robust to intensity differences. In a second part of this work, we propose a new approach that overcomes some of the main limitations of the existing techniques while complying with most of the desired requirements above. Our algorithm combines the mathematical framework for computing a variational flow proposed by Hermosillo et al. [G. Hermosillo, C. Chefd'Hotel, O. Faugeras, A variational approach to multi-modal image matching, Tech. Rep., INRIA (February 2001).] with the radial lesion growth pattern presented by Bach et al. [M. Bach Cuadra, C. Pollo, A. Bardera, O. Cuisenaire, J.-G. Villemure, J.-Ph. Thiran, Atlas-based segmentation of pathological MR brain images using a model of lesion growth, IEEE Trans. Med. Imag. 23 (10) (2004) 1301-1314.]. Results on patients with a meningioma are visually assessed and compared to those obtained with the most similar method from the state-ofthe-art.
\end{abstract}

(c) 2006 Elsevier Ireland Ltd. All rights reserved.

\section{Introduction}

Atlas-based segmentation is a widely used technique for automatic segmentation of normal MR images. It relies on the existence of a reference image volume (called atlas) in which structures of interest have been carefully segmented, usually by hand. To segment a new image volume, a transformation that registers (i.e., puts in point-to-point correspondence) the atlas to this volume is first computed. By point-to-point correspondence we refer to a spatial correspondence. This trans-

\footnotetext{
* Corresponding author. Tel.: +41 21693 4712; fax:+41 216937600.

E-mail addresses: meritxell.bach@epfl.ch (M. Bach Cuadra), decraene@tele.ucl.ac.be (M. De Craene), valerie.duay@epfl.ch (V. Duay), macq@tele.ucl.ac.be (B. Macq), claudio.pollo@chuv.hospvd.ch (C. Pollo), jp.thiran@epfl.ch (J.-Ph. Thiran).

URL: http://lts5www.epfl.ch/, www.tele.ucl.ac.be/, http://lts5www.epfl.ch/, www.tele.ucl.ac.be, http://lts5www.epfl.ch/. 0169-2607/\$ - see front matter @ 2006 Elsevier Ireland Ltd. All rights reserved.

doi:10.1016/j.cmpb.2006.08.003
} 
formation is then used to project labels assigned to structures from the atlas onto the image volume to be segmented. Thus, the segmentation problem is reduced to a registration problem that tried to capture and compensate the normal anatomical variability.

Unfortunately, atlas-based segmentation has been of limited use in presence of large space-occupying lesions. In fact, brain deformations induced by such lesions may dramatically shift and deform functionally important brain structures. Fig. 1 shows some examples of lesions that might largely deform brain structures. Here, the goal of the registration becomes even more complex: it not only tries to capture the normal anatomical variability between subjects but also the deformation induced by the pathology. Moreover, the anatomical meaningful correspondence assumption done in the atlas-based segmentation paradigm is usually strongly violated since voxels located inside the damaged area have no correspondence to the atlas. However, precise segmentation of functionally important brain structures would provide useful information for therapeutic consideration of space-occupying lesions, including surgical, radio-surgical, and radiotherapeutic planning, in order to increase treatment efficiency and minimize neurological damage.

In this work, we focus on the problem of inter-subject registration of MR images with large tumors, inducing a significant shift of surrounding anatomical structures. First, we review the existing methods that have been proposed to deal with this problem. This brief survey introduces the discussion about the requirements or desirable properties that we consider necessary to be fulfilled by a registration method in this context. Second, we propose a new approach that overcomes some of the main limitations of the existing techniques while complying with most of the desired requirements.

The remaining of the paper is organized as follows. First, a brief review of the state-of-the-art methods and a discussion of their main limitations are presented in Section 2. Then, the data set used in this work is introduced in Section 3. The proposed method is presented and validated in Section 4. Finally, Sections 5 and 6 conclude this work with a discussion and future research lines.

\section{Survey of registration methods for brain MR images with tumors}

Two early works related to atlas-based segmentation in presence of space-occupying tumors were published in the late 1990s. Kyriacou and Davatzikos [3] proposed a biomechanical model of the brain using a finite-element method. They first model soft tissue deformations induced by the tumor growth and, then, they registered the anatomical atlas with a transformed patient image from which the tumor was removed. On the other hand, Dawant et al. [4] relied on a simpler approach based on optical-flow-Thirion's demons algorithm [5]-for both tumor growth modeling and atlas matching deformation. Their solution was called seeded atlas deformation (SAD), as they put a seed with the same intensity properties as the lesion in the atlas image, and then computed the non-rigid registration. More recently, Bach et al. and Polio et al. [2,6], presented an improved seeded atlas deformation algorithm: instead of applying the nonlinear registration algorithm to the whole image, a specific model of tumor growth (MLG) inside the tumor area was proposed, which assumed the tumor growth radial from a single voxel seed. Demons algorithm [5] was used outside the tumor area and the displacement vector field was regularized by an adaptive Gaussian filter to avoid possible discontinuities. This approach overcame some of the limitations of SAD method but other ones arisen. For instance, the placement of the seed needed expertise and presegmentation of the lesion was necessary. Also, since the demons algorithm used mean squared difference of intensities as similarity measure, contrast agent induced some errors in the deformation field.

Other methods [7-9] locally adapted the elasticity of the transformation, rather than modeling the deformation induced by the tumor, in a way that large deformations induced by the tumor can be captured. To avoid tumor segmentation, Duay et al. in [7] did not use a special scheme to preserve the regularity of the transformation in the areas where the images differ topologically (e.g., inside the tumor). Thus, they allowed the deformation might contain some singularities inside the lesion. They proposed to use an elasticity map related to the atlas to allow larger displacements over regions (the inside of the lateral ventricles) that are known

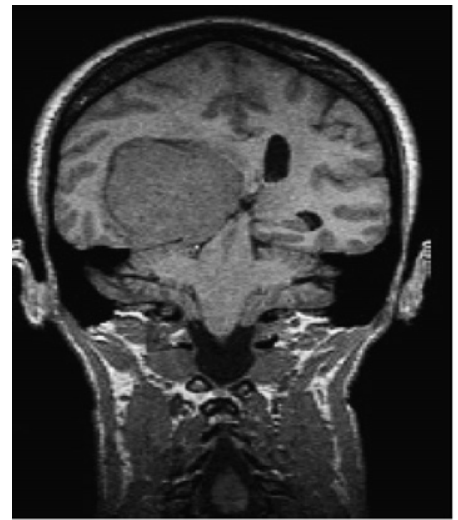

(a)

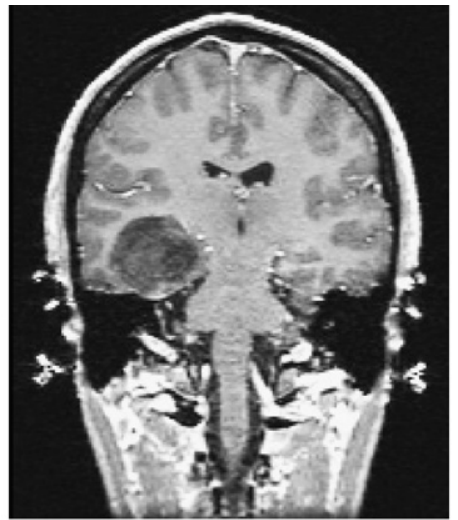

(b)

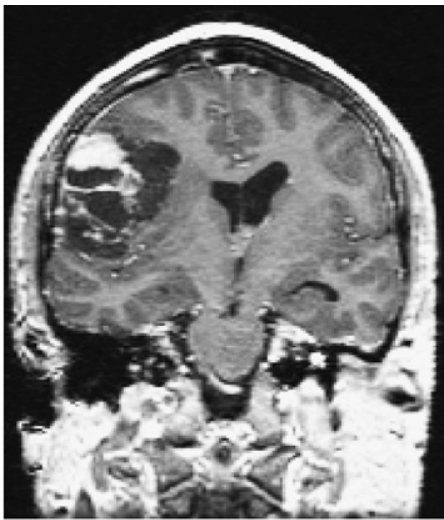

(c)

Fig. 1 - Some examples of brain lesions. (a) Meningioma, (b) glioma, and (c) astrocy-toma. 
to be very sensitive to the tumor deformation. On the contrary, in order to prevent bad deformations inside the lesion area, Stefanescu proposed in $[8,9]$ to impose more rigidity on the tumor. This required the previous segmentation of the patient's tumor. Similarly, in [10], Liu and Davatzikos used a statistically-based interpolation method to correct their deformation in the areas with low confidence (around the tumor region).

Recently, Nowinski et al. [11] proposed to use a Talairach registration followed by a three-dimensional (3D) nonlinear tumor deformation based on a geometric assumption, as in [2], that the tumor compresses its surrounding tissues radially. A more sophisticated model of lesion growth was proposed by Mohamed and Davatzikos in [12] based on 3D biomechanical finite element model. Then, in [13], they combine this model (by atlas seeding) with a deformable registration based on the so-called HAMMER algorithm [14,15].

This brief survey is summarized in Table 1 under three criteria: type of registration, model of lesion and main limitations. Let us analyze here after the state-of-the-art methods regarding each criteria.

A large variety of registration approaches are proposed for atlas-based segmentation methods of pathological brain images: biomechanical models, parametric deformations with basis functions, surface to surface registration, Talairach warping, demons algorithm, and pair-and-smooth methods. It is difficult to determine which of them is better suited for our problem of interest. But we agree with Stefanescu [9] that having a dense deformation field guarantees the transformation to be represented at a maximal level of detail in the whole image space, thus having a point-to-point spatial correspon- dence. Note that by point-to-point correspondence, we refer to the fact that the transformation does not have any singularity due to the lack of anatomical correspondence (for instance points that come from nowhere since the atlas does not have a tumor). Of course, regularity of the deformation field should be ensured.

Most of the methods either explicitly model the lesion growth and seed the atlas with a lesion or, on the contrary, ignore the information contained within the lesion. On one side, a seeding strategy associated with a tumor growth model ensures the continuity of the transformation in the tumor area and preserves from any irregularities that could appear in this region due to the lack of anatomical correspondence between atlas and patient. However, this usually involves an increment in the number of parameters to estimate, like location and size of the seed or tumor. On the other side, the methods that do not consider the information within the lesion locally adapt the elasticity of the transformation in a way that large deformations induced by the tumor can be captured even without modeling the lesion growth. Moreover, modeling areas or structures with different deformability allow to obtain a better accuracy of the segmented contours since real deformations are usually inhomogeneous.

Main limitations are more specific to the registration method and similarity measure. For instance, biomechanical methods or surfaced-based methods need also the presegmentation of some functionally important brain structures of the patient image. In our opinion, it is desirable to avoid presegmentation since segmentation-based registration algorithms are usually very sensitive to presegmentation accuracy and reproducibility. However, most of the state-of-the-art

Table 1 - State-of-the-art of atlas-based segmentation methods for pathological brain images with large space-occupying lesions

\begin{tabular}{|c|c|c|c|}
\hline Author & Registration & Lesion & Limitations \\
\hline Kyriacou and Davatzikos [3] & $\begin{array}{l}\text { Biomechanical model } \\
\text { Finite elements }\end{array}$ & Remove lesion from patient & $\begin{array}{l}\text { 2D implementation } \\
\text { Presegmentation } \\
\text { Tumor segmentation }\end{array}$ \\
\hline Dawant et al. [4] & Demons & Seeded atlas deformation & $\begin{array}{l}\text { Mask of information } \\
\text { Too much deformability } \\
\text { Tumor segmentation }\end{array}$ \\
\hline Bach et al. [2], Pollo et al. [6] & $\begin{array}{l}\text { Demons } \\
\text { Model of lesion growth }\end{array}$ & $\begin{array}{l}\text { Seeded atlas } \\
\text { Radial growing } \\
\text { One-voxel seed }\end{array}$ & $\begin{array}{l}\text { Contrast agent } \\
\text { Tumor segmentation } \\
\text { Seed position }\end{array}$ \\
\hline Duay et al. [7] & $\begin{array}{l}\text { Parametric registration } \\
\text { Elasticity locally adapted }\end{array}$ & No model & Estimation of elasticity parameters \\
\hline Stefanescu et al. $[8,9]$ & $\begin{array}{l}\text { Pair-smooth registration } \\
\text { Elasticity locally adapted }\end{array}$ & $\begin{array}{l}\text { No model } \\
\text { Interpolation }\end{array}$ & $\begin{array}{l}\text { Prior information } \\
\text { Tumor segmentation }\end{array}$ \\
\hline Liu et al. [10] & $\begin{array}{l}\text { Surface to surface } \\
\text { Volumetric registration }\end{array}$ & $\begin{array}{l}\text { No model } \\
\text { Interpolation }\end{array}$ & $\begin{array}{l}\text { Cortical surface only } \\
\text { Tumor segmentation }\end{array}$ \\
\hline Nowinski and Belov [11] & $\begin{array}{l}\text { Hybrid landmark } \\
\text { Talairach warping }\end{array}$ & Remove lesion from patient & $\begin{array}{l}\text { Talairach landmarks } \\
\text { Brain hull } \\
\text { Tumor segmentation }\end{array}$ \\
\hline \multirow{2}{*}{$\begin{array}{l}\text { Mohamed et al. [13], } \\
\text { Zacharaki et al. [15] }\end{array}$} & Biomechanical model & Mass effect of tumor growth & Skull-stripped \\
\hline & Hierarchical attribute registration & & $\begin{array}{l}\text { Segmented images } \\
\text { Parameter estimation }\end{array}$ \\
\hline
\end{tabular}


methods need to segment at least the lesion (excepting [7]). While this could be a limitation in some complex cases with infiltrating tumors or presence of edema, there exist automated methods that allow an accurate segmentation of a large range of lesions [16-18] (see [19] for a recent review of brain tumor segmentation algorithms).

Voxel-based methods naturally avoid the problem of presegmenting functionally important brain structures (excepting the lesion) since they directly work on voxel intensities. In atlas-based segmentation applications, it is worth to use such feature because choosing the atlas the same modality as the patient simplifies the registration problem. However, other confounding effects may appear in a mono-modal registration. For instance, due to different acquisition parameters or acquisition devices, same structures might appear with different intensities or a bias field might corrupt the image. Moreover, in the case of pathological MR brain images, edema, or contrast agent are often present. Thus, a similarity measure robust to intensity changes is desirable too.

In our opinion, a significant drawback of voxel-based methods is that the requirement of a smooth deformation field-necessary for preserving the topology of the anatomical structures-limits the accuracy of the resulting segmentation. A widely used solution is to allow globally or locally more elasticity to the deformation in order to obtain more local deformation (see for instance $[2,4,7,8]$ ) with the risk of increasing the irregularity of the deformation field and thus of the contours. Moreover, this does not ensure that the sought level of precision will be obtained. To cope with this problem, more local constraints have to be included in the atlas registration process. These constraints should permit the registration on relevant structures, to impose the smoothness of the contours and to introduce more prior knowledge such as the intensity distribution or the admissible shapes of the objects selected to drive the registration.

In conclusion, we consider that the main requirements and desirable properties of a registration algorithm for atlas-based segmentation with large-space occupying lesions are:

(1) To have a dense deformation field in the whole image.

(2) To obtain high level of structure segmentation accuracy.

(3) To encompass a model of deformations induced by the tumor.
(4) To avoid tedious patient presegmentation.

(5) To be robust to intensity differences (for instance due to the contrast agent).

We propose in Section 4, a dense deformation field estimation algorithm for pathological MR brain images registration. It combines the mathematical framework for computing a variational flow (here, from Mutual Information similarity metric) proposed by Hermosillo et al. [1] with the pattern presented by Bach et al. [2] where an atlas is seeded with a tumor seed and its growth is assumed to be radial to the lesion contour. Thus, our approach fulfills requirements 1, 4, and 5. An adaptive Gaussian filter is used for regularization. This partially fulfills requirement 3 since different deformability is allowed not for different anatomical structures but for three regions of the image (inside the lesion area, close to the lesion and the rest of the brain). Currently, requirement 2 is not included in the algorithm but we discuss its possible implementation in Section 5 .

\section{Data set}

The patient images used in this study have been retrieved from the Surgical Planning Laboratory (SPL) of the Harvard Medical School \& NSG Brain Tumor Database [20]. They consist of three SPGR T1-weighted volumes of 124 coronal slices of 256 pixels $\times 256$ pixels and $0.9375 \mathrm{~mm} \times 0.9375 \mathrm{~mm} \times 1.5 \mathrm{~mm}$ of voxel size. All of them present a meningioma of similar size: $43 \mathrm{~mm} \times 50 \mathrm{~mm} \times 37 \mathrm{~mm}, 41 \mathrm{~mm} \times 42 \mathrm{~mm} \times 52 \mathrm{~mm}$, and $36 \mathrm{~mm} \times 37 \mathrm{~mm} \times 40 \mathrm{~mm}$. Only patients with meningiomas are presented here. In fact, considering all the possible spaceoccupying lesions in a unified framework is almost impossible. We selected meningiomas because they are treated with radio surgery or stereotactic radiotherapy, where an atlas-based segmentation of sensitive structures of the brain is of importance. This kind of tumor is usually benign and its extracerebral growth usually induce a pure shift and deformation of the underlying brain structures (see patient MR images on Fig. 2). Moreover, no brain edema was observed on the data set. Notice that all patient images have been acquired using a contrast agent (gadolinium).

The digital atlas used in this work comes from the SPL [21]. It is composed of two images: An MRI and a label image. The

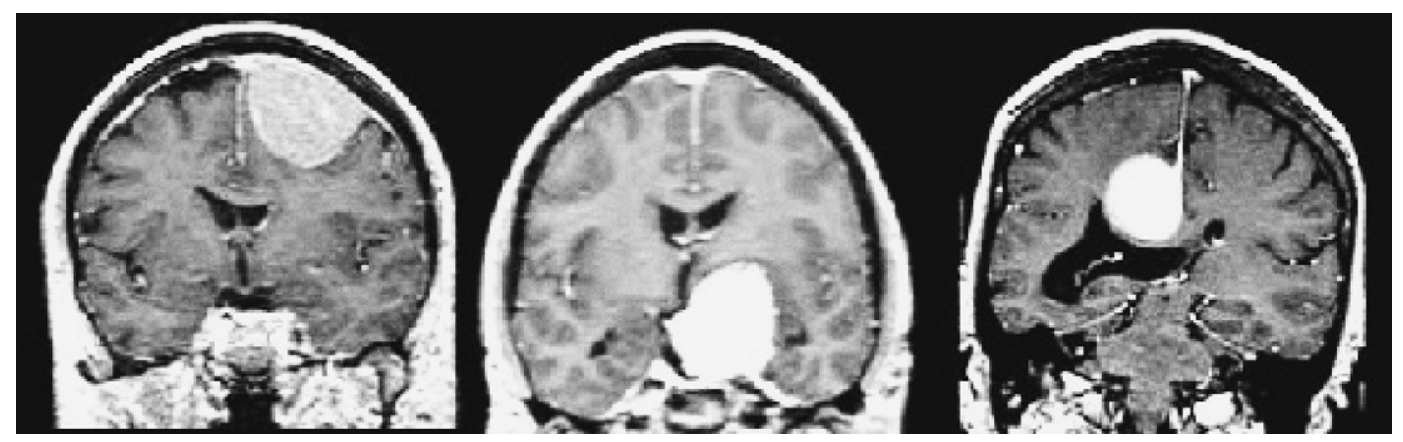

Fig. 2 - SPGR T1-weighted patients with meningioma. Contrast agent is gadolinium. Location and size is (from left to right): left frontal of $43 \mathrm{~mm} \times 50 \mathrm{~mm} \times 37 \mathrm{~mm}$, left parsellar of $41 \mathrm{~mm} \times 42 \mathrm{~mm} \times 52 \mathrm{~mm}$, and right parietal of $36 \mathrm{~mm} \times 37 \mathrm{~mm} \times 40 \mathrm{~mm}$. 


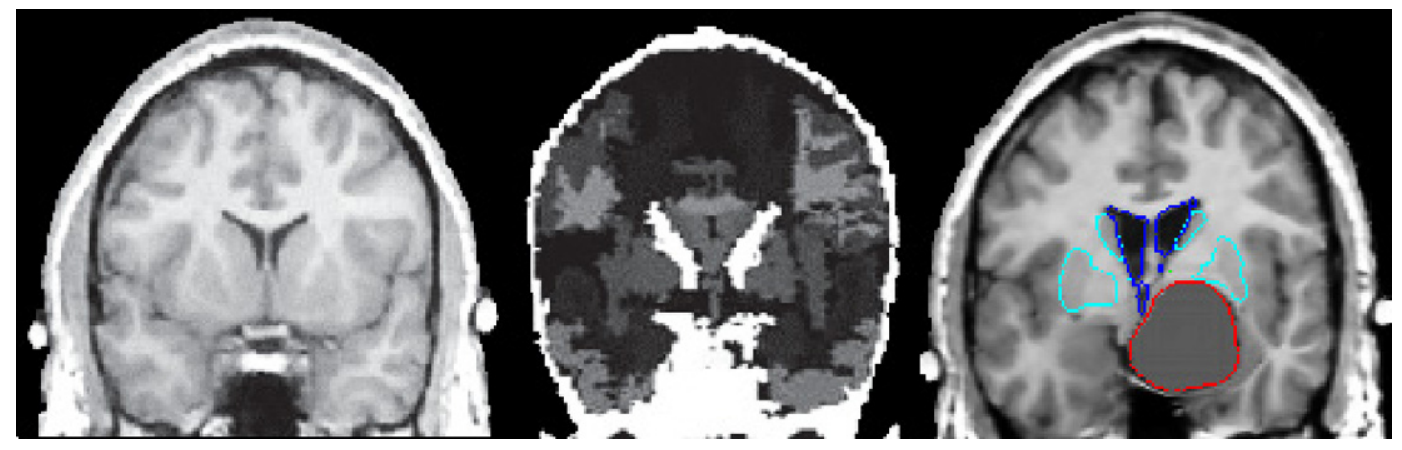

Fig. 3 - Digitized atlas. Left and central panel respectively are T1-weighted MR and labels from the surgical panning laboratory (SPL) of the Harvard Medical School [21]. Right panel shows the deformed atlas (MR and labels) after MI flow.

MRI has been made of MR data from a single normal subject scanned with high resolution $256 \times 256 \times 160$ volume data set in coronal orientation with $0.9375 \mathrm{~mm} \times 0.9375 \mathrm{~mm} \times 1.5 \mathrm{~mm}$ voxel size (Fig. 3 left)). The label atlas image contains anatomical and functional structures that have been manually segmented (Fig. 3 center).

\section{Mutual information flow registration}

The approach proposed in this work is a non-rigid registration method that combines a mutual information flow and a radial lesion growth model [22]. This approach is in fact based on the works of $[1,2]$.

\subsection{Introduction}

Optical flow has been widely used in the context of atlas-based segmentation $[2,4,5]$. The classical optical flow formulation relies on the assumption that the intensity distributions are identical in the fixed and moving images. Even if both images are from the same modality (MR in our case), a contrast product is often used in clinical practice to better appreciate the anatomical limits of tumors. In these cases, methods that rely on similarity measures based on intensity differences would fail because large deformations will be computed in areas where the contrast agent is present. Here, instead of using the least squares minimization criteria, a mutual information (MI) flow method that maximizes the mutual information is proposed to overcome this limitation. That makes the deformation more robust in regions where a contrast product is present.

Our registration method follows the theory presented by Hermosillo et al. [1] where a mathematical framework for computing a variational flow from various similarity metrics (mean square error, mutual information, correlation ratio) was introduced. The flow computed from global similarity measures (as shown in the next section for the case of mutual information) incorporates local information (moving image gradient at this point) as well as global information (marginal and joint probability distributions of signal intensities).

\subsection{Mutual Information flow registration for normal subjects}

For compensating inter-subject deformations, a variational expression of mutual information has been introduced by Hermosillo et al. [1]. If $p^{f, m}$ denotes the joint probability density between the fixed and the moving image and $p^{f}\left(p^{m}\right)$ denotes the marginal density of the fixed (moving) image signal intensities ( $f$ and $m$ denote the signal intensity functions of the fixed and the moving image respectively), mutual information is computed for a displacement field $u(x)$ following

$$
\begin{aligned}
\mathrm{MI}_{u}= & H(F)+H_{u}(M)-H_{u}(F, M)=-\sum_{i_{1}} p^{f}\left(i_{1}\right) \log \left(p^{f}\left(i_{1}\right)\right) \\
& -\sum_{i_{2}} p_{u}^{m}\left(i_{2}\right) \log \left(p_{u}^{m}\left(i_{2}\right)\right)+\sum_{i_{1}, i_{2}} p_{u}^{f, m}\left(i_{1}, i_{2}\right) \log \left(p_{u}^{f, m}\left(i_{1}, i_{2}\right)\right),
\end{aligned}
$$

where $H(F)$ designate the fixed image signal intensity entropy, $H_{u}(M)$ the moving image signal intensity entropy and $H_{u}(F$, $M)$ the joint entropy between fixed and moving images signal intensities. The displacement field $u$ is defined from the fixed to the moving image domain and influences the moving and joint image intensities distributions. The dynamic range of the fixed and moving image intensities is divided into bins of constant sizes. In Eq. (1), $i_{1}$ and $i_{2}$ stand for the intensities bin indexes in the fixed ( $i_{1}$ index) and the moving ( $i_{2}$ index) images.

A variational flow of this metric is obtained by a first order perturbation analysis. If $h(x)$ is defined as a continuous perturbation function ${ }^{1}$ multiplied by a scalar factor $\epsilon$, the optimal displacement field $u(x)$ is defined as the function vanishing the first variation of the similarity metric regarding $\epsilon$

$\left.\frac{\partial \mathrm{MI}_{u_{\mathrm{opt}}+\epsilon h}}{\partial \in}\right|_{\epsilon=0}=0$.

In the mutual information case, the left member of (2) can be expressed as (see Thevenaz et al. [23] for details about the

\footnotetext{
${ }^{1}$ For detailed conditions on this perturbation function, see [1].
} 
computation of this criterion's derivative)

$$
\left.\frac{\partial \mathrm{MI}_{u+\in h}}{\partial \in}\right|_{\epsilon=0}=\left[\sum_{i_{1}, i_{2}} \frac{\partial p_{u+\in h}^{f, m}\left(i_{1}, i_{2}\right)}{\partial \in} \log \left(\frac{p_{u+\in h}^{f, m}\left(i_{1}, i_{2}\right)}{p_{u+\in h}^{m}\left(i_{2}\right)}\right)\right]_{\epsilon=0} .
$$

In our implementation, the joint probability distribution is estimated as suggested by Mattes [24] using a twodimensional (2D) B-Spline kernel. A third-order B-Spline in the moving image dimension ensures a well defined derivative regarding $\epsilon$

$p_{u}^{f, m}\left(i_{1}, i_{2}\right)=\frac{1}{|\Omega|} \int_{\Omega} \beta^{1}\left(f(x)-i_{1}\right) \beta^{3}\left(m(x+u)-i_{2}\right) d x$,

where $\beta^{j}$ designates a 1D B-Spline function of order $j$.

Injecting the definition of $p^{f m}$ (Eq. (4)) in Eq. (3) yields

$\left.\frac{\partial \mathrm{MI}_{\mathrm{u}+\epsilon h}}{\partial \in}\right|_{\epsilon=0}=\int_{\Omega} \kappa_{u}^{f, m}(x) \nabla m(x+u) h(x) \mathrm{d} x>\nabla_{u} \mathrm{MI}, h(x)>$

where $\kappa_{u}^{f, m}$ is defined by

$\kappa_{u}^{f, m}(x)=\frac{1}{|\Omega|} \sum_{i_{1}, i_{2}} \log \left(\frac{p_{u}^{f, m}\left(i_{1}, i_{2}\right)}{p_{u}^{m}\left(i_{2}\right)}\right) \beta^{1}\left(f(x)-i_{1}\right) \beta^{3^{\prime}}\left(m(x+u)-i_{2}\right)$.

Eq. (5) shows that the derivative regarding the $\in$ parameter in Eq. (2) is the scalar product between two functions: $h(x)$ (the perturbation function) and the MI metric flow defined by

$\nabla_{u} \mathrm{MI}=\kappa_{u}^{f, m}(x) \nabla m(x+u)$.

This means that for vanishing the first moment of the metric (left member in Eq. (2)) for any choice of perturbation $h$, the metric flow has to tend to a zero vector field for the optimal displacement field $u$. A conventional way of reaching this objective is to iteratively update the current displacement field by a term proportional to the metric flow defined in Eq. (7). The optimal displacement field $u$ in the sense of a first-order variational analysis will be reached once the update tends to zero.

\subsection{Atlas to pathological brain non-rigid registration}

Our approach to brain atlas deformation in the presence of space occupying tumors follows the same steps as proposed by Bach et al. [2] but differs from the fact that a flow optimizing mutual information is used instead of the demons algorithm [5]. The overall process can be summarized as follows:

(1) An affine transformation [25] is applied to the atlas image in order to globally match the patient.

(2) The lesion is segmented using the adaptive template moderated spatially varying statistical classification (ATM SVC) algorithm [16].
(3) The atlas is manually seeded by an expert with a singlevoxel placed on the estimated origin of the patient's lesion.

(4) The non-linear registration based on a mutual information flow and a MLG tumor growth model is performed in order to deform the seeded atlas on the target patient.

The non-rigid deformation method we propose distinguishes between those two areas fixed from the lesion segmentation. Outside the lesion, the MI flow force as defined in Eq. (7) is applied. Inside the lesion, the tumor growth model assumes a radial growth of the tumor from the tumor seed. The displacement vector computed at every voxel using either the MI flow force or the tumor growth model is regularized by an adaptive Gaussian filter to avoid discontinuities around the lesion borders. Three areas are considered in the smoothing process: Inside the lesion area, close to the lesion (within $10 \mathrm{~mm}$ of the tumor) where large deformations occur, and the rest of the brain. Smoothing is not necessary inside the lesion because the vector field induced by the model of lesion growth is highly regular and the continuity is ensured. Different amount of elasticity is denoted by the standard deviation of the Gaussian filter $\sigma$. So, ( $\sigma=0$ inside the lesion area. In the region close to the tumor (including the tumor contour) there are large deformations due to the tumor growth. Then, it is necessary to allow large elasticity, i.e., $\sigma$ should have a small value, typically $0.5 \mathrm{~mm}$. In the rest of the brain, deformations are smaller, due primarily to inter-patient anatomical variability. So, a larger $\sigma$ proves to be better, as it simulates a more rigid transformation $(\sigma=1)$.

\subsection{Results}

The SPL digital atlas MR image has been deformed on three patients of the data set using the algorithm described above. At each iteration, the joint histogram is estimated by random sampling through the image domain. The mutual information flow is then computed at each voxel using Eq. (7). The number of iterations is fixed and remains a parameter of the algorithm to be chosen by the user. The implementation of the mutual information flow has been integrated in the Insight Toolkit [26] as a subclass of the PDEDe formable registration function class. This implementation of MI flow is publically made available in [27].

Fig. 3 shows the atlas MR and labels before and after the MI flow deformation for patient 2. Fig. 4 shows the atlas contours of the ventricles, the central nuclei, the thalamus and the tumor projected after transformation on all patients (dark blue, light blue, green, and red, respectively). A 3D rendering of these segmentations is shown in Fig. 5. Visually assessment does not show any significant difference between optical and mutual information flow. However, the two flows behave differently in the presence of different contrasts in both images. For instance, around the mid-sagittal plane, the classical optical flow is disturbed by a high difference in signal intensities (the mid-sagittal plane is bright in the patient image and dark in the atlas). This is illustrated on Fig. 6: Fig. 6(a) shows a coronal slice of the target patient image, the deformed atlas image is shown in (b) using optical flow and in (c) using MI flow. This illustrates a main limitation of OF (and of methods that use the SSD as similarity measure) since the assumption 


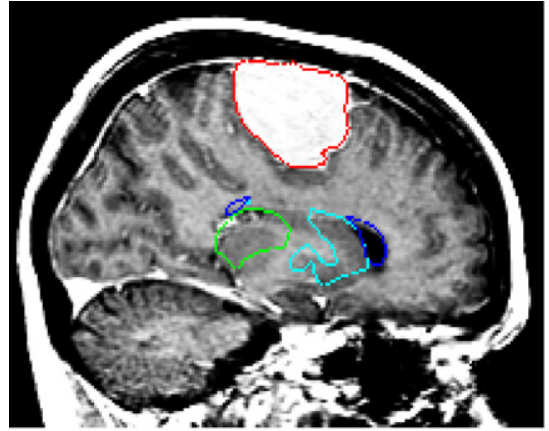

Case 1

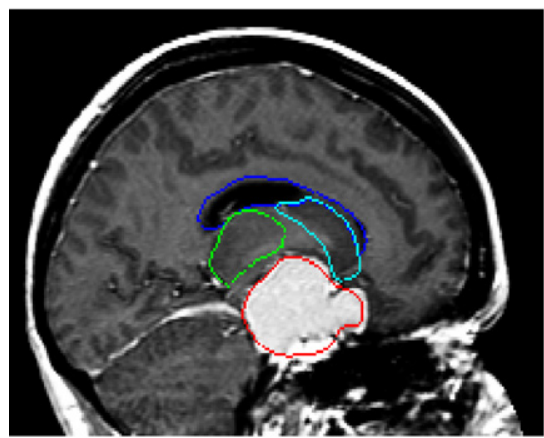

Case 2

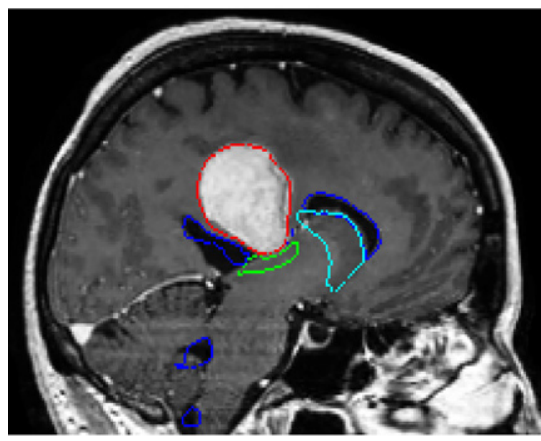

Case 3
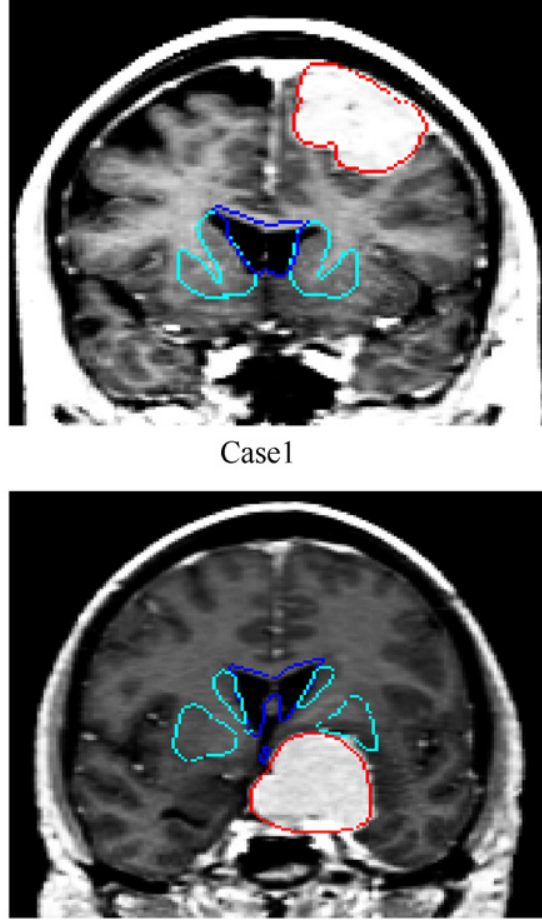

Case2

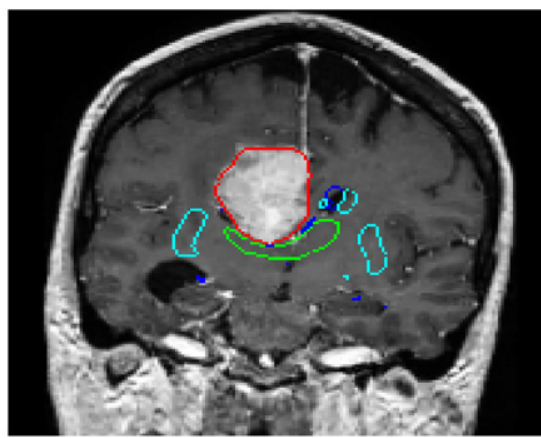

Case 3

Fig. 4 - Segmentation results obtained after alignment of the atlas on three patients with large tumors. Contours of the tumor, ventricles, thalamus, and central nuclei are overlayed on the patient image. A radial tumor growth deformation model is used inside the lesion. Outside the lesion, a mutual information variational flow is used to compensate inter-subject variations.

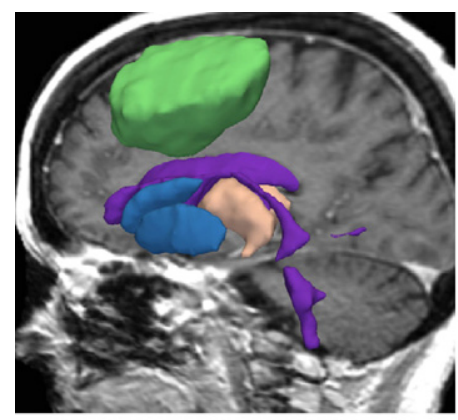

Case 1

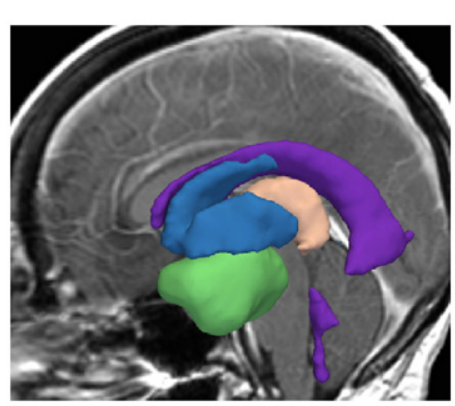

Case 2

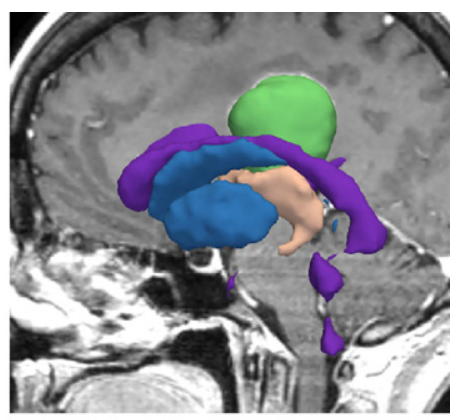

Case 3

Fig. 5 - 3D rendering of tumor (in green), ventricles (in mauve), thalamus (in beige), and central nuclei (in blue) from the images in Fig. 4. (a) Patient image, (b) optical flow (Demons), and (c) mutual information flow. (For interpretation of the references to color in this figure legend, the reader is referred to the web version of this article.) 


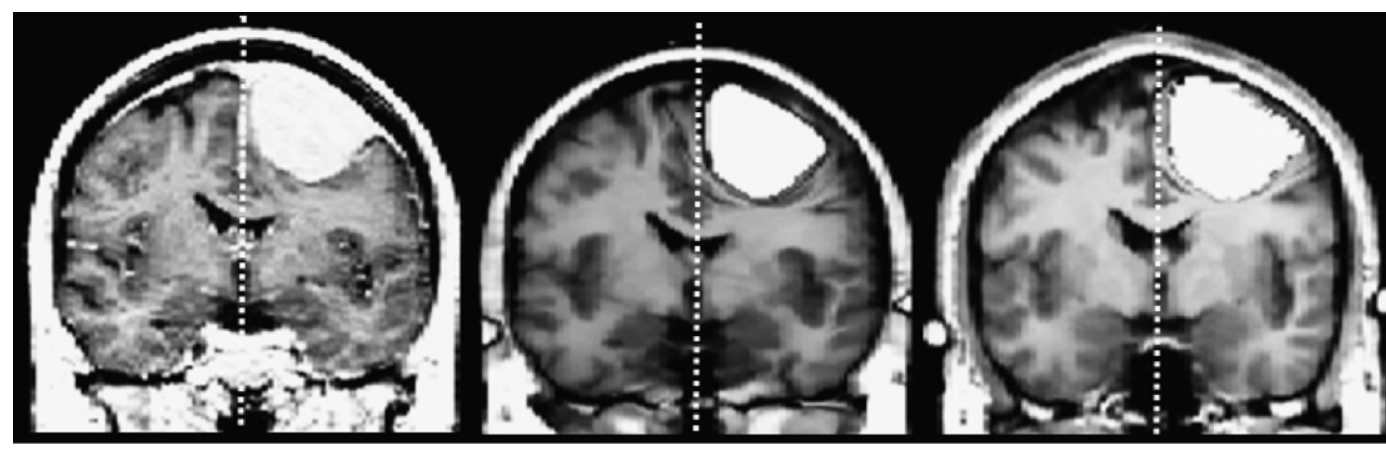

(a) Patient image

(b)

Optical flow (Demons)

(c) Mutual information flow

Fig. 6 - Robustness of the MI flow to intensity differences along the midsagital plane (dotted line). The patient target image is shown in (a). Sub-figure (b) and (c) show registration results using optical (b), and MI (c) flows.

of intensity correspondence between anatomical structures is violated because of the contrast agent. On the contrary, the MI flow proves to be robust to these intensity changes and the deformation at the mid sagittal plane is not misguided. In fact, robustness of MI criteria against intensity differences is one of its main characteristics and the main reason of its success as originally presented in $[28,29]$. Intuitively, MI maximization captures the best alignment by attempting to reduce the entropy in the joint space of fixed and moving image intensities. This joint entropy can be interpreted as a measure of the dispersion in the joint histogram. Even if a structure has a different intensity in the two modalities, bringing this structure into alignment will create a cluster in the joint histogram and will reduce entropy.

\subsection{Validation using a synthetic patient}

In this section, performances of the mutual information and optical flows are compared quantitatively. For this purpose, a synthetic healthy patient (as proposed in [2]) was created. The basis of this idea is to avoid as much as possible the inter-subject variability, thus, the algorithm should ideally be applied to the patient before the growth of the lesion but of course this information is not available. Then, a good estimation of how the patient brain was before the appearance of the tumor is needed. We know that the brain has approximately symmetrical structures. Therefore, the damaged hemisphere was almost like the healthy one before the tumor growth. Once the symmetry plane is found, a simple mirroring of the healthy side is done to generate a new synthetic healthy patient-atlas.
Note that, with this new atlas, the most similar brain a posteriori to the healthy brain of the patient is obtained.

The algorithm described in Section 4 is then run to match this synthetic healthy patient-atlas on the real patient. This has been done for Patient 1. Results are then compared to those obtained with an optical flow force like described in [2]. For doing this, the STAPLE [30] algorithm is run on both $\mathrm{MI}$ and OF segmentations. This way, a reference segmentation is obtained and it is compared to the results of both methods. Note that, in this context, the use of the STAPLE does not assess how good a method is since no manual segmentations are available but it quantifies how similar both OF and MI flow performances are.

The performance matrix (its diagonal corresponds to the specificity and sensitivity parameters) with respect to the reference segmentation is given in Table 2. No significant difference can be observed between the performances of MI and optical flows. The diagonal coefficients (giving the probability of true positive decisions for each label) show that the segmentation of the thalamus has better performances with the MI flow while optical flow has a slightly better accuracy on the central nuclei segmentation.

\section{Discussion}

In this paper we have presented a MI flow algorithm combined with a radial growth model that allow us to estimate a dense deformation field in the whole image. This approach is in fact very similar to the one presented in [2] but it solves one of

Table 2 - STAPLE performance matrix for automatic atlas-base segmentations using optical flow and mutual information flow

\begin{tabular}{|c|c|c|c|c|c|c|c|c|}
\hline \multirow[t]{2}{*}{ Truth } & \multicolumn{4}{|c|}{ Optical flow } & \multicolumn{4}{|c|}{ MI flow } \\
\hline & Background & Central nuclei & Ventricles & Thalamus & Background & Central nuclei & Ventricles & Thalamus \\
\hline Background & 0.9997 & 0.0899 & 0.0607 & 0.0527 & 0.9997 & 0.0981 & 0.0605 & 0.0316 \\
\hline Central nuclei & 0.00012 & 0.9001 & 0.0098 & 0.0019 & 0.0001 & 0.8850 & 0.0031 & 0.0 \\
\hline Ventricles & 0.00011 & 0.00999 & 0.92432 & 0.0180 & 0.0001 & 0.0151 & 0.9253 & 0.0116 \\
\hline Thalamus & 0.00003 & 0.0 & 0.00523 & 0.9274 & 0.0001 & 0.0018 & 0.0111 & 0.9568 \\
\hline
\end{tabular}

Diagonal coefficients are the probability of true positive decisions for each label. 
its major limitations. This has been shown in Fig. 6 where the contrast product present in the midsagittal plane induced non-realistic deformations in [2] while here, as expected, the MI flow algorithm has proven to be robust to intensity differences. Regarding the segmentation accuracy of key structures, no other significant differences have been observed between MI flow and optical flow algorithm (see Table 2). These results are very encouraging but not yet sufficiently since contours lack of precision in some places (see segmented ventricles of case 3 in Fig. 4).

The main hypotheses of the lesion growth model used here are that the lesion expands radially and that there is no infiltration and no edema. Therefore, only meningiomas have been considered. The simplicity of this model is both its weakness and strength. Other-potentially more complex-models of growth and infiltration should obviously be considered for other types of tumors and lesions, but this is beyond the scope of this paper.

Another limitation of this approach is shared by all voxelbased methods since they often lead to a compromise between the accuracy of the resulting segmentation and the smoothness of the transformation. Here, the regularization of the deformation field is done by an adaptive Gaussian filtering similarly to the one proposed in [2]. Three different elasticities (see Section 4.3) are allowed according to the distance to the tumor: inside the tumor the radial force ensures the regularity, near the tumor large deformability is allowed and far from the lesion elasticity modeling normal anatomy deformations is applied. A more realistic model of deformability would be to consider not only the distance to the tumor but also the implicit elasticity of some structures such the ventricles, midsagittal plane or skull. This could be included as proposed by Duay et al. [7] by considering that different anatomical structures in the atlas have different elasticity parameters $\sigma$ (the estimation and adjustment for each patient of this elasticity parameters remaining a difficult task). However, even if a better deformability is modeled, in certain places, contours will not be placed accurately enough when using a voxel-based method due to the regularization step.

To cope with this problem, more local constraints have to be included in the atlas registration process (see Section 2, requirement 2) for instance by incorporating local statistical measures in the registration process as proposed recently by Commowick et al. in [31]. In our opinion, among the different techniques proposed so far for image analysis, the active contour framework is particularly well suited to define and implement local constraints but it was designed for image segmentation only. Recently, Duay et al. [32,33] presented an active contour framework for both segmentation and registration. In there, a local-non rigid registration in a level-set framework is presented along with some preliminary but promising results on pathological brain image registration. Such a framework allows not only to easily include local constraints but also to select different elasticities for different structures in the atlas and it does not need from presegmentation of the tumor. Like the biomechanical methods, this algorithm can also be seen as a surface-based registration method. Its first difference is that it computes the deformation based on the image and not on mechanical or biological laws. This implies that its accuracy does not depend on a good physical mod- eling of the tissues. Above all, its main advantage is that it does not need a presegmentation of the patient image. The contours defined in the atlas evolve following an energy functional specially defined to be minimal when they have reached the desired object contours, as in active contour-based segmentation framework [34]. Unfortunately, the main limitation of the surface to surface registration algorithm remains. As the deformation is only based on contours of interest, the probability of registration errors increases more we are far from these contours. As far as we know, most of the existing methods for registration of images with space-occupying tumors are either surface-based $[10,12,15,35]$ or voxel-based $[2,4,6-8,36]$ approaches. However, in our opinion it is worth to study how to combine the advantages of both approaches.

In a future work, we would be interested to study a two steps approach by combining the MI flow segmentation results as initial step of an active contour segmentation-registration approach [33]. Moreover, the method should be tested on more data sets containing different kind and size of lesions in order to better validate. Also, it would be important to study a case where the lesion evolution is known to see if the model of lesion growth we have proposed is near or far from the reality.

\section{Conclusion}

In this paper, we studied the application related to atlas-based segmentation in presence of space-occupying tumors. In a first part of this document we reviewed the existing methods that has been proposed to deal with this problem and we defined the requirements that we consider necessary to be fulfilled by a registration method in this context: Having a dense and smooth deformation field to ensure a point-to-point correspondence, to be able to model different deformability for some structures, to be robust to intensity differences and to be able to include local prior knowledge. The final contribution of this work is a new algorithm based on a MI flow associated a model of lesion growth. This algorithm overcomes the main limitations of existing methods while fulfilling most of the requirements defined above.

\section{Acknowledgments}

Our acknowledgment goes to Dr Ron Kikinis who has provided us with the Surgical Planning Laboratory (SPL) atlas and also to Dr Simon Warfield for providing us the STAPLE algorithm. This work is supported by the Center for Biomedical Imaging (CIBM) of the Geneva-Lausanne Universities, the EPFL, and the foundations Leenaards and Louis-Jeantet, by the Belgian Walloon Region through a First Europe Convention (EPI A320501R049F/415732), by the Swiss National Science Foundation under grant number 205320-101621 and by the SIMILAR Network of Excellence.

\section{REFERENCES}

[1] G. Hermosillo, C. Chefd'Hotel, O. Faugeras, A variational approach to multi-modal image matching, Tech. rep., INRIA (February 2001). 
[2] M. Bach Cuadra, C. Polio, A. Bardera, O. Cuisenaire, J.-G. Villemure, J.-Ph. Thiran, Atlas-based segmentation of pathological MR brain images using a model of lesion growth, IEEE Trans. Med. Imag. 23 (10) (2004) 1301-1314.

[3] S.K. Kyriacou, C. Davatzikos, Nonlinear elastic registration of brain images with tumor pathology using a biomechanical model, IEEE Trans. Med. Imag. 18 (7) (1999) 580-592.

[4] B.M. Dawant, S.L. Hartmann, S. Gadamsetty, Brain atlas deformation in the presence of large space-occupying tumors, in: Proceedings of the Second Medical Image Computing and Computer-Assisted Intervention (MICCAI), 19-22 September, 1999, Cambridge, UK, pp. 589-596.

[5] J.-P. Thirion, Image matching as a diffusion process: An analogy with Maxwell's demons, Med. Image Anal. 2 (3) (1998) 243-260.

[6] C. Polio, M. Bach Cuadra, O. Cuisenaire, J.-G. Villemure, J.-Ph. Thiran, Segmentation of brain structures in presence of a space-occupying lesion, Neuroimage 24 (4) (2005) 990-996.

[7] V. Duay, P. DHaese, R. Li, B. Dawant, Non-rigid registration algorithm with spatially varying stiffness properties, in: Proceedings of the IEEE International Symposium on Biomedical Imaging (ISBI): From Nano to Macro, 15-18 April, 2004, Arlington, VA, USA, pp. 408-411.

[8] R. Stefanescu, O. Commowick, G. Malandain, P.-Y. Bondiau, N. Ayache, and X. Pennec, Non-rigid atlas to subject registration with pathologies for conformal brain radiotherapy, in: Proceedings of the Medical Image Computing and Computer-Assisted Intervention (MICCAI), 26-29 September, 2004, Saint-Malo, France, pp. 704-711.

[9] R. Stefanescu, Parallel nonlinear registration of medical images with a priori information on anatomy and pathology, Thése de sciences, Université de Nice-Sophia-Antipolis, 2005.

[10] T. Liu, D. Shen, C. Davatzikos, Deformable Registration of Tumor-Diseased Brain Images, in: Proceedings of the Medical Image Computing and Computer-Assisted Intervention (MICCAI), September 26-29, 2004, Saint-Malo, France, pp. 720-728.

[11] W.L. Nowinski, D. Belov, Toward atlas-assisted automatic interpretation of MRI morphological brain scans in the presence of tumor, Acad. Radiol. 12 (8) (2005) 1049-1057.

[12] A. Mohamed, C. Davatzikos, finite element modeling of brain tumor mass-effect from $3 d$ medical images, in: Proceedings of the Medical Image Computing and Computer-Assisted Intervention (MICCAI), 26-29 October, 2005, Palm Springs, Ca, USA, pp. 400-408.

[13] A. Mohamed, D. Shen, C. Davatzikos, deformable registration of brain tumor images via a statistical model of tumor-induced deformation, in: Proceedings of the Medical Image Computing and Computer-Assisted Intervention (MICCAI), 26-29 October, 2005, Palm Springs, Ca, USA, pp. 263-270.

[14] D. Shen, C. Davatzikos, HAMMER: Heirarchical attribute matching mechanism for elastic registration, IEEE Trans. Med. Imag. 21 (11) (2002) 1421-1439.

[15] E. Zacharaki, D. Shen, A. Mohamed, C. Davatzikos, Registration of brain images with tumors: Towards the construction of statistical atlases for therapy planning, in: Proceedings of the IEEE International Symposium on Biomedical Imaging (ISBI), 6-9 April, 2006, Arlington, Virginia, USA, in press.

[16] S.K. Warfield, M. Kaus, F.A. Jolesz, R. Kikinis, Adaptive, template moderated, spatially varying statistical classification, Med. Image Anal. 4 (1) (2000) 43-55.

[17] M. Prastawa, E. Bullitt, S. Ho, G. Gerig, A brain tumor segmentation framework based on outlier detection, Med. Image Anal. 8 (3) (2004) 275-283.

[18] S. Ho, E. Bullitt, G. Gerig, Level-Set Evolution with Region Competition: Automatic 3-D Segmentation of Brain Tumors, in: Proceedings of the sixth International Conference on Pattern Recognition (ICPR'02), 11-15 August, 2002, Quebec, Canada, p. 10532.

[19] M. Schmidt, Automatic brain tumor segmentation, Master of science, University of Alberta, Canada, 2005.

[20] M. Kaus, S.K. Warfield, A. Nabavi, P.M. Black, F.A. Jolesz, R. Kikinis, Automated segmentation of MRI of brain tumors 218 (2) (2001) 586-591.

[21] R. Kikinis, et al. A digital brain atlas for surgical planning, model driven segmentation and teaching, IEEE Trans. Visual. Comput. Graph. 2 (3) (1996).

[22] Mathieu De Craene, Dense deformation estimation for pairwise and multi-subjects registration, Doctorat en sciences appliquées, Université Catholique de Louvain (UCL), Belgium, October 2005.

[23] P. Thevenaz, M. Unser. Optimization of mutual information for multiresolution image registration, IEEE Trans. Image Process. 9 (12) (2000).

[24] D. Mattes, D.R. Haynor, H. Vesselle, T.K. Lewellen, W. Eubank, PET-CT image registration in the chest using free-form deformations, IEEE Trans. Med. Imag. 22 (1) (2003) 120-128.

[25] O. Cuisenaire, J.-P. Thiran, B. Macq, C. Michel, A. D. Voider, F. Marques, Automatic Registration of 3D MR images with a Computerized Brain Atlas, Proc. SPIE Med. Imag. 1719 (1996) 438-449.

[26] http://www.itk.org.

[27] M. De Craene, A. du Bois d'Aische, B. Macq, S. Warfield, Incorporating metric flows and sparse jacobian transformations in itk, The Insight Journal. URL http://hdl.handle.net/1926/183.

[28] P. Viola, W.M. Wells III, Alignment by maximization of mutual information, in: Proceedings of the Fifth International Conference on Computer Vision, 20-23 June, 1995, Cambridge, Massachusetts, USA, pp. 16-23.

[29] F. Maes, et al., Multimodality image registration by maximization of mutual information, IEEE Trans. Med. Imag. 161 (2) (1997) 187-198.

[30] S.K. Warfield, K.H. Zou, W.M. Wells, Simultaneous truth and performance level estimation (STAPLE): An algorithm for the validation of image segmentation, IEEE Trans. Med. Imag. 23 (7) (2004) 903-921

[31] O. Commowick et al., Incorporating statistical measures of anatomical variability in atlas-to-subject registration for conformal brain radiotherapy, in: Proceedings of Medical Image Computing and Computer-Assisted Intervention (MICCAI), vol. 2, 2005, pp. 927-934.

[32] V. Duay, N. Houhou, J.-Ph. Thiran, Atlas-based segmentation of medical images locally constrained by level sets, in: Proceedings of the International Conference in Image Processing (ICIP), vol. 2, 11-14 September, 2005, Genova, Italy, pp. 1286-1289.

[33] V. Duay, M. Bach Cuadra, X. Bresson, J.-Ph. Thiran, Dense deformation field estimation for atlas registration using the active contour framework, in: Proceedings of the European Signal Processing Conference (EUSIPCO), 4-6 September, 2006, Florence, Italy, in press.

[34] S. Osher, J.A. Sethian, Fronts propagating with curvature-dependent speed-algorithms based on Hamilton-Jacobi formulations, J. Comput. Phys. 79 (1) (1988) 12-49.

[35] D. Kyriacou, C. Davatzikos, A biomechanical model of soft tissue deformation with applications to non-rigid registration of brain images with tumor pathology, in: Proceedings of the MICCAI '98, 11-13 October, 1998, Cambridge, MA, USA, pp. 531-538.

[36] P.Y. Bondiau, et al., Atlas-based automatic segmentation of MR images: Validation study on the brainstem in radiotherapy context, Int. J. Radiat. Oncol. Biol. Phys. 61 (1) (2005) 289-298. 